\title{
La Suisse a besoin d'une loi stricte sur les produits du tabac
}

\author{
Jacques Cornuz \\ Prof. Dr, directeur Policlinique médicale universitaire de Lausanne
}

La Journée mondiale sans tabac du 31 mai sera placée sous le thème du commerce illicite. D’après l'Organisation mondiale de la santé, une cigarette sur dix est vendue illégalement. La nouvelle loi sur les produits du tabac représente une chance de renforcer la prévention contre le tabagisme au niveau légal. Les conséquences néfastes du tabagisme sur la santé justifient l'établissement de règles strictes dans le domaine du commerce des produits du tabac, l'objectif étant d'améliorer la santé dans notre pays.

Cinquante ans après la parution aux Etats-Unis du premier rapport du Surgeon General (1964), un nouveau rapport présente une liste de nouvelles pathologies liées au tabac, parmi lesquelles figurent le diabète de type 2, la polyarthrite rhumatoïde et le carcinome hépatocellulaire. Rappelons qu'un fumeur sur deux meurt de son tabagisme: une "roulette russe» qui n'aurait pas une balle, mais trois! Les conséquences fatales du tabagisme justifient de soumettre le commerce des produits du tabac à des restrictions sévères.

L'avant-projet de la loi fédérale sur les produits du tabac recèle des lacunes qui entravent sa cohérence avec la prévention du tabagisme. Des mesures supplémentaires sont indispensables, car les conditions-cadres légales ne peuvent avoir une influence décisive sur la consommation de tabac que si elles en limitent le commerce. La santé de la population prime les intérêts économiques de l'industrie du tabac.

Correspondance:

Prof. Dr Jacques Cornuz Policlinique médicale universitaire de Lausanne Rue du Bugnon 44 CH-1011 Lausanne Tél. 0213144732 Fax 0213146099 www.pmu-lausanne.ch

Leffet de la publicité en faveur du tabac, notammen sur les jeunes, est aujourd'hui incontesté. Or, les mesures proposées par le Conseil fédéral dans le domaine du commerce des produits du tabac ont une portée insuffisante. La nouvelle loi devrait notamment interdire la publicité dans les points de vente et l'incitation à l'achat par l'octroi de rabais.

\section{Cigarettes électroniques}

L'avant-projet du Conseil fédéral autorise désormais la vente de cigarettes électroniques contenant de la nicotine, ce qui est en concordance avec un récent rapport d'experts suisses [1]. Mais il faut dès lors que l'accès soit aussi limité que pour la cigarette de tabac. Par conséquent, les cigarettes électroniques, qu'elles contiennent ou non de la nicotine, devraient être soumises aux mêmes règlementations que les autres produits du tabac, y compris en ce qui a trait à la publicité, à la promotion et au parrainage.

Sur l'échelle Tobacco Control Scale 2013 in Europe, publiée par l'Association des ligues européennes de lutte contre le cancer, la Suisse n'obtient que 45 points sur 100, principalement parce qu'une limitation globale du commerce lui fait défaut. La nouvelle loi sur les produits du tabac représente une occasion unique pour la Suisse de rattraper son retard en matière de règlementation dans ce domaine.

\section{Journée mondiale sans tabac}

Le commerce illicite affaiblit les mesures de prévention du tabagisme. C'est le message principal que veut faire passer la Journée mondiale sans tabac en 2015. Les cigarettes vendues au noir sont bon marché, ce qui incite surtout les jeunes à la consommation, tout en privant les Etats d'importantes recettes fiscales. En 2012, I'UE a estimé que la contrebande de cigarettes faisait perdre chaque année plus de dix milliards d'euros en droits de douane à l'institution et à ses membres. Malgré cette constatation, le Conseil fédéral a renoncé à introduire dans son avant-projet de loi des mesures contre le commerce illicite. Si la Suisse se tient à l'écart, ce vide juridique pourrait se révéler problématique dans la collaboration internationale des douanes et des corps de police.

\section{Informations}

- Sur le site web de l'Association suisse pour la prévention du tabagisme, une rubrique est consacrée à la loi sur les produits du tabac: www.at-suisse.ch $\rightarrow$ Facts $\rightarrow$ Loi sur les produits du tabac

- Le site www.observatoire-marketing-tabac.ch documente toutes les formes de publicité en faveur des produits du tabac, de promotion et de parrainage présentes en Suisse. 